\title{
Multi-wavelength observations of filament oscillations induced by shock waves
}

\author{
Yuandeng Shen \\ Yunnan Observatories, Chinese Academy of Sciences, Kunming 650011, China \\ email: ydshen@ynao.ac.cn
}

\begin{abstract}
Two cases of filament oscillations induced by large-scale coronal shock waves are presented. For the first case, a chain of transverse oscillating filaments are observed in a proper order after the passing of a shock wave, and it is found that the they were triggered by the surface component of the dome-shaped shock wave. For the second case, simultaneous transverse oscillation of a limb prominence and longitudinal oscillation in an on-disk filament are launched by a single shock wave. It is found that the interaction angle between the shock wave and the prominence axis is the key to launch transverse or longitudinal filament oscillations. In addition, filament magnetic fields are estimated, using the measured parameters.
\end{abstract}

Keywords. Shock Waves, Filaments, Oscillations, Prominences.

\section{Introduction}

Large amplitude filament (prominence) oscillations could be classified into transverse and longitudinal oscillations (Arregui et al. 2012, Tripathi et al. 2009). Previous studies indicate that the former type is often trigger by shock waves such as chromosphere Moreton and coronal extreme-ultraviolet waves (e.g., Ramsey \& Smith 1966, Eto et al. 2002, Okamoto et al. 2004, Gilbert et al. 2008, Shen et al. 2014a, 2014b), while the latter is usually associated with nearby micro flare, filament, and jet activities (e.g., Jing et al. 2003, 2006, Vršnak et al. 2007, Li \& Zhang 2012). The velocity amplitude, period, and damping time of transverse oscillations are in ranges of $6-41 \mathrm{~km} \mathrm{~s}^{-1}, 11-29$, and 25 - 180 minutes, while those for longitudinal oscillations are $30-100 \mathrm{~km} \mathrm{~s}^{-1}, 44-160$, and 115 - 600 minutes, respectively. Theoretical studies of filament oscillations can be found in Kleczek \& Kuperus 1969, Hyder 1966, and Luna \& Karpen 2012. So far, the driving mechanism of oscillating filaments and their relationship between shock waves are still unclear. Here, two cases of filament oscillation cases are presented to diagnose the properties of the oscillating filaments and the associated coronal shock waves.

\section{Results}

The first case was on September 06, 2011. Four successive winking (oscillating) filaments (F1-F4) and a micro jet are observed right after the X2.1 flare in AR11283, and their start times are obviously depending on their distances to the flare (see Figure 1. (a)). In EUV observations taken by the Atmospheric Imaging Assembly (AIA) onboard the Solar Dynamics Observatory (SDO), a bright shock wave is observed in the northwest of AR11283, which composed of two components: a fast $\left(850 \mathrm{~km} \mathrm{~s}^{-1}\right)$ and a slow (542 $\mathrm{km} \mathrm{s}^{-1}$ ) shock waves. By comparing the start time of filament oscillations and the position of the shock wave speeds, it is found that the oscillating filaments are associated with the slow component. F1 is taken as an example to show the filament oscillation. One can see the obvious periodically appearance and disappearance of the filament at the red- and blue-wings, indicating the downward and upward motions of the filament. 


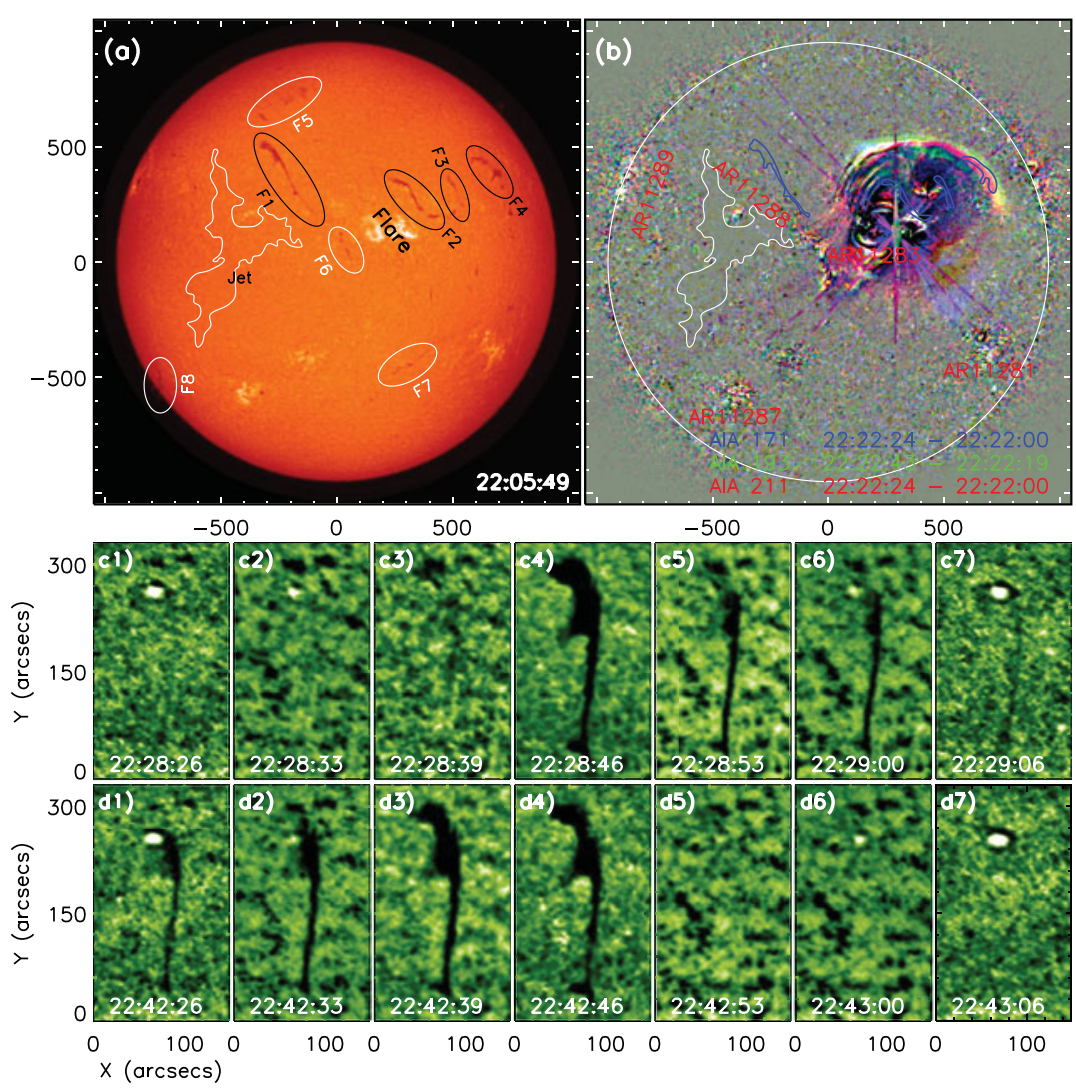

Figure 1. The first case. a, SMART $\mathrm{H} \alpha$ center image. b, running difference tri-color image made from AIA 171, 193, and $211 \AA$ A images. c and d, SMART H $\alpha$ Doppler images. From left to right, they are $-1.2,-0.8,-0.5,0.0,+0.5,+0.8$, and $+1.2 \AA$ images shifting from the $\mathrm{H} \alpha$ center.

The periods, velocity amplitudes, and damping times of the oscillating filaments are in the ranges of 11-22 minutes, 6-14 $\mathrm{km} \mathrm{s}^{-1}$, and 25-60 minutes, respectively. Using these parameters, it is estimated that the radial component magnetic field of the oscillating filaments are in the range of 5-10 Gauss.

The second case was on August 09, 2011. An X6.9 flare occurred in AR11263, which launched an impulsive coronal shock wave that triggered the transverse oscillation of a prominence (P) and the longitudinal oscillation of a filament (F2). Figure 2. (a)-(d) show the condition before the flare. In the time-distance plot (Figure 2. (e)), one can see that the prominence start to oscillate immediately after the passing of the shock wave. It is measured that the oscillation period of the prominence is 13.5 minutes. Large amplitude longitudinal oscillation was observed in F2 after the passing of the shock wave (see Figure 2. (f)), whose oscillation period is about 80.2 minutes. Using the measured parameters, it is obtained that the radial component magnetic field of the prominence and the poloidal field of the filament are about 8 and 3 Gauss, respectively.

A simple scenario is proposed to explain the different oscillation patterns observed in the prominence and the filament (see Figure 2. $(\mathrm{g})$ and $(\mathrm{h})$ ). It is propose that the orientation of a filament or prominence relative to the normal vector of the incoming shock wave should be an important factor for launching transverse or longitudinal filament oscillations. i.e., if the wavefront is perpendicular (parallel) to a filament axis, transverse (longitudinal) oscillations could be expect in the filament. 

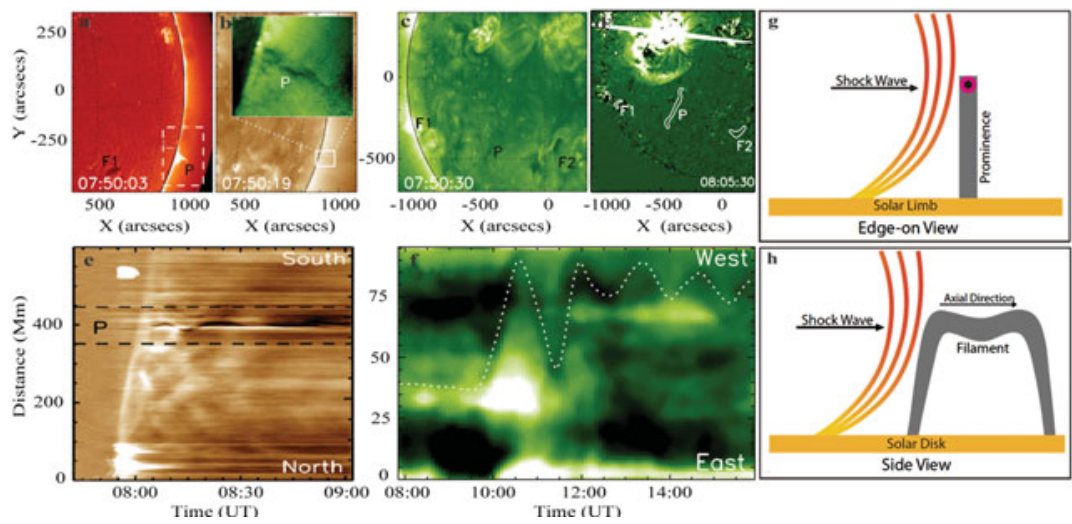

Figure 2. The second case. a, SMART H $\alpha$ image. b, AIA $193 \AA$ image, c and d, STEREO $193 \AA$ direct and running-difference images, respectively. e (f), time-distance plot made from AIA (STEREO) 193 (195) Å observations along a line perpendicular (parallel) to the prominence axis. P, F1, and F2 denote the prominence and the two filaments. $\mathrm{g}$ and $\mathrm{h}$, cartoon model demonstrating the driving mechanism of oscillating filaments.

\section{Discussions}

According to Veronig et al. (2010), a coronal shock wave usually has a dome shape in the upward direction, and on the lateral one can observe another surface shock wave that has a relative lower speed. For the first case, the fast wave should be the dome part, while the slow one should be the lateral surface wave. Therefore, considering the relationship between the start time of the oscillating filaments and the speeds of the shock waves, it is proposed that the chains of filament oscillations are triggered by the slow wave component. In summary, it is conclude that coronal shock waves is a good agent for triggering and connecting successive but separated solar activities in the solar atmosphere. They can not only efficiently launch filament oscillations and other solar eruptive activities, but also important for diagnosing filament and the surrounding coronal parameters.

\section{Acknowledgements}

This work is supported by Chinese foundations (11403097, 2015FB191), and the Youth Innovation Promotion Association (2014047).

\section{References}

Arregui, I., Oliver, R., \& Baluster, J. L. 2012, Living Rev. Sol. Phys., 9, 2

Eto, S., Isobe, H., Narukage, N., Asai, A., \& Morimoto, T., et al. 2002, PASJ, 54, 481

Gilbert, H. R., Daou, A. G., Young, D., Tripathi, D., \& Alexander, D. 2008, ApJ, 685, 629

Hyder, C. L. 1966, Z. Astrophys., 63, 78

Jing, J., Lee, J., Spirock, T. J., Xu, Y., \& Wang, H., et al. 2003, ApJ, 584, L103

Jing, J., Lee, J., Spirock, T. J., \& Wang, H. 2006, Sol. Phys., 236, 97

Kleczek, J. \& Kuperus, M. 1969, Sol. Phys. 6, 72

Li, T. \& Zhang, J. 2012, ApJ, 760, L10

Luna, M. \& Karpen, J. 2012, ApJ, 750, L1

Okamoto, T. J., Nakai, H., Keiyama, A., Narukage, N., \& UeNo, S., et al. 2004, ApJ, 608, 1124

Ramsey, H. E. \& Smith, S. F. 1966, AJ, 71, 197

Shen, Y., Ichimoto, K., Ishii, T. T., Tian, Z., \& Zhao, R., et al. 2014a, ApJ, 786, 151

Shen, Y., Liu, Y. D., Chen, P. F., \& Ichimoto, K. 2014b, ApJ, 795, 130

Tripathi, D., Isobe, H., \& Jain, R. 2009, Space Sci. Rev., 149, 283

Veronig, A. M., Muhr, N., Kienreich, I. W., Temmer, M., \& Vršnak, B. 2010, ApJ, 716, L57

Vrs̆nak, B., Veronig, A. M., Thalmann, J. K, \& Z̆ic, T. 2007, A\& A, 471, 295 